\title{
Solar Review: Characterizing Thin Film Photovoltaics
}

\author{
K. Dovidenko*, J. Cournoyer*, D. Vernooy*, B. Lita**, F. Seymour** \\ * GE Global Research Center, Niskayuna, NY 12309 \\ ** PrimeStar Solar, Arvada, CO 80004
}

Materials characterization plays critical role in the development of new materials and technologies. In this paper, we review recent worldwide progress in solar CdTe and CIGS thin film technologies including device background, materials challenges and corresponding approaches to characterization, with particular emphasis on probing at nano-scale. Significant characterization breakthroughs will be discussed as well as the most challenging aspect of each materials system.

The two highly successful thin film solar technologies are CdTe utilizing $1.5 \mathrm{eV}$ direct optical bandgap of $\mathrm{CdTe}$, and copper indium gallium (di)selenade (CIGS), where the absorbing semiconductor consists of $\mathrm{CuIn}_{\mathrm{x}} \mathrm{Ga}_{(1-\mathrm{x})} \mathrm{Se}_{2}$ with the bandgap continuously varied as a function of $\mathrm{Ga}$ content between $1 \mathrm{eV}(\mathrm{x}=1)$ and $1.7 \mathrm{eV}(\mathrm{x}=0)$. Record cells of $16.5 \%$ efficiency $\mathrm{CdTe}$ and $19.9 \%$ CIGS have been reported by NREL $[1,2]$.

Challenges to device development are related to CdTe and CIGS materials challenges and to the required technological approaches for cell fabrication involving multiple steps that cause numerous interconnected effects. State-of-the-art thin film solar cells have complicated structures consisting of stacks of several different semiconductor and metallic layers, multiple interfaces. To fully understand the materials, processing and reliability aspects of the device, these multilayered thin film heterostructures require a broad range of characterization approaches, including those at nano-scale. Focused ion beam and scanning electron microscopy (SEM) provide information regarding the film thickness, grain morphology, potential device integrity/delamination or shunting issues. SEM combined with energy dispersive x-ray analysis or wavelength dispersive spectroscopy and electron backscatter diffraction give composition and orientation information, respectively. Transmission electron microscopy and electron energy loss spectroscopy are the ultimate techniques for high spatial resolution analysis of interfaces and grain boundaries. A number of precise compositional studies are being carried out by electron probe microanalysis and X-ray fluorescence techniques. Although high efficiency CIGS devices can be made with wide tolerances to variations in $\mathrm{Cu}(\mathrm{InGa}) \mathrm{Se}_{2}$ composition, it is highly important to monitor the $\mathrm{Cu} /(\mathrm{In}+\mathrm{Ga})$ ratio, as well as the $\mathrm{Ga} /(\mathrm{In}+\mathrm{Ga})$ ratio [3]. Techniques such as Auger electron spectroscopy, x-ray photoelectron spectroscopy (XPS) and secondary ion mass spectrometry (SIMS, dynamic and time-of-flight) provide information on the distribution of elements as a function of depth with different spatial resolution, sensitivity and analysis area. True chemical bonding information on surfaces subjected to different processing conditions and treatments can be obtained through XPS. One of the challenges for characterizing both CdTe and CIGS system is the strong susceptibility of these materials to the electron/ion beam-induced 
radiation damage, introducing the extra characterization challenges that continually need resolution [4]. Those challenges are exaggerated by using small probes with high beam current that are often needed for nano-scale analysis.

One of the critical components of both CdTe- and CuInGaSe-based solar cells is typically the cadmium sulphide layer. An example of a FIB cross-section through the CdTe-based device is shown in Fig. 1. Thin - from a few tens to a hundred nm - CdS film is commonly used as a window layer in CdTe solar technology. The CdS is mainly explored for its heterojunction forming capability with the p-type cadmium telluride and p-type copper indium diselenide. The role of $\mathrm{CdS}$ in $\mathrm{CdTe}$-based cells still remains controversial after decades of extensive research. Minimizing the CdS thickness in CdTe cells allows conformal coverage of the substrate without forming shunting defects. For CIGS, the CdS layer can be kept somewhat thicker to avoid shunting. A variety of deposition methods can be used to fabricate the CdS layers, resulting in different electrical properties and crystallographic phases (hexagonal, cubic or mixture of those). Despite a large number of studies that have been devoted to $\mathrm{CdS}$, questions remain about the internal structure of these thin films, particularly when they are buried under the other device layers. New x-ray diffraction techniques are underway to study these. CdTe/CdS interfacial mixing in CdTe-based cells is a subject of numerous complex studies as it is believed to define the location of the heterojunction. The task is not trivial since depth profiling techniques such as XPS and SIMS often lack the needed interfacial resolution due to sample surface roughness and extreme development of the latter during sputter depth profiling. We will review the results from all traditional materials characterization techniques along with more direct heterojunction observation methods like electron beam induced current.

[1] X. Wu et al., Proc. of 17th E-PVSEC (2001) 995

[2] M. A. Repins et al., Progress in Photovoltaics: Research and Applications, 16(3) (2008) 235-239

[3] W. N. Shafarman and L. Stolt, "Handbook of Photovoltaic Science and Engineering", Edited by Antonio Luque and Steven Hegedus, John Wiley \& Sons (2003)

[4] K. Dovidenko et al., Microscopy and Microanalysis, 15 (2009) 1386

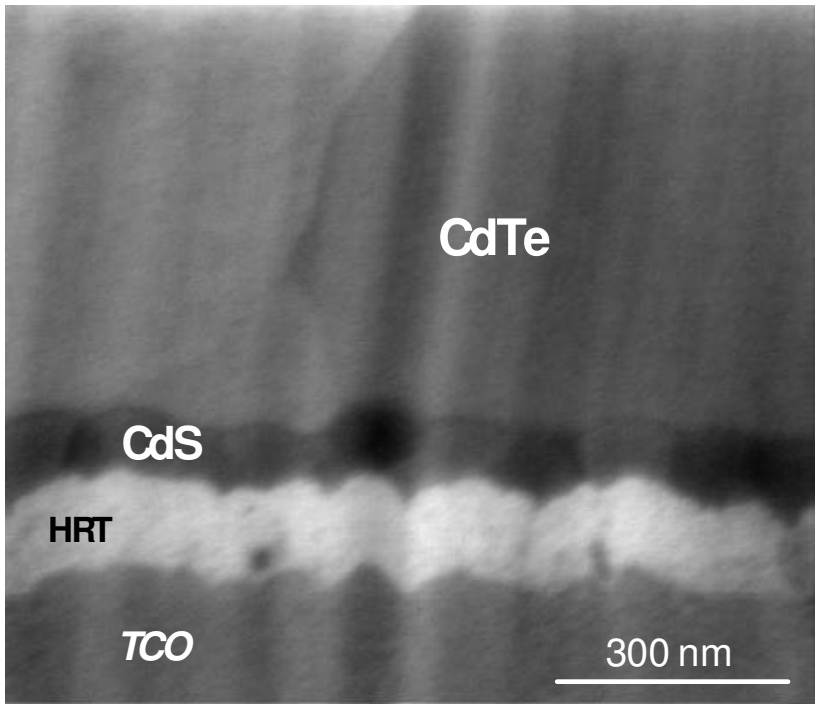

Fig. 1

CdTe-based cell: secondary electron image of a cross-section made by Focused Ion Beam. 